\title{
Binary frequency of planet-host stars at wide separations
}

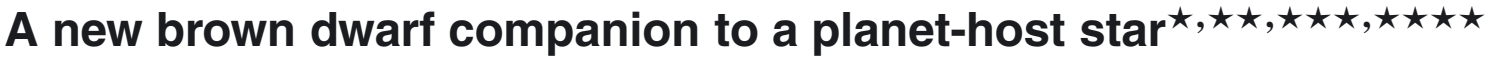

\author{
N. Lodieu ${ }^{1,2}$, A. Pérez-Garrido ${ }^{3}$, V. J. S. Béjar ${ }^{1,2}$, B. Gauza ${ }^{1}$, M. T. Ruiz ${ }^{4}$, R. Rebolo ${ }^{1,2,5}$, \\ D. J. Pinfield ${ }^{6}$, and E. L. Martín ${ }^{7}$
}

1 Instituto de Astrofísica de Canarias (IAC), Calle Vía Láctea s/n, 38200 La Laguna, Tenerife, Spain
e-mail: [nlodieu; vbejar; bgauza; rrl] @iac.es
2 Departamento de Astrofísica, Universidad de La Laguna (ULL), 38206 La Laguna, Tenerife, Spain
3 Universidad Politécnica de Cartagena, Campus Muralla del Mar, Cartagena, 30202 Murcia, Spain
4 Departamento de Astronomía, Universidad de Chile, Casilla 36-D, Santiago, Chile
5 Consejo Superior de Investigaciones Científicas, CSIC, Spain
6 Centre for Astrophysics Research, Science and Technology Research Institute, University of Hertfordshire, Hatfield AL10 9 AB,
UK
7 Centro de Astrobiología (INTA-CSIC), Departamento de Astrofísica, PO Box 78, 28691 Villanueva de la Cañada, Madrid, Spain

Received 14 May 2014 / Accepted 4 August 2014

\section{ABSTRACT}

\begin{abstract}
Aims. The aim of the project is to improve our knowledge on the multiplicity of planet-host stars at wide physical separations. Methods. We cross-matched approximately 6200 square degree area of the southern sky imaged by the Visible Infrared Survey Telescope for Astronomy (VISTA) Hemisphere Survey (VHS) with the Two Micron All Sky Survey (2MASS) to look for wide common proper motion companions to known planet-host stars. We complemented our astrometric search with photometric criteria. Results. We confirmed spectroscopically the co-moving nature of seven sources out of 16 companion candidates and discarded eight, while the remaining one stays as a candidate. Among these new wide companions to planet-host stars, we discovered a T4.5 dwarf companion at 6.3 arcmin ( 9000 au) from HIP 70849, a K7V star which hosts a 9 Jupiter mass planet with an eccentric orbit. We also report two new stellar $\mathrm{M}$ dwarf companions to one $\mathrm{G}$ and one metal-rich $\mathrm{K}$ star. We infer stellar and substellar binary frequencies for our complete sample of 37 targets of $5.4 \pm 3.8 \%$ and $2.7 \pm 2.7 \%$ ( $1 \sigma$ confidence level), respectively, for projected physical separations larger than $\sim 60-160$ au assuming the range of distances of planet-host stars $(24-75 \mathrm{pc})$. These values are comparable to the frequencies of non planet-host stars. We find that the period-eccentricity trend holds with a lack of multiple systems with planets at large eccentricities $(e>0.2)$ for periods less than 40 days. However, the lack of planets more massive than 2.5 Jupiter masses and short periods ( $<40$ days) orbiting single stars is not so obvious due to recent discoveries by ground-based transit surveys and space missions.
\end{abstract}

Key words. stars: low-mass - brown dwarfs - techniques: photometric - techniques: spectroscopic - surveys

\section{Introduction}

The discoveries of the first extrasolar planet orbiting a solartype star and the first brown dwarfs were announced the same year (Mayor \& Queloz 1995; Rebolo et al. 1995; Nakajima et al. 1995). Since then, more than one thousand exoplanets have been discovered, mainly thanks to radial velocity surveys and transit campaigns but also through direct imaging, microlensing, and timing ${ }^{1}$. In the meantime, a similar number of nearby field L, T, and Y dwarfs $(\sim 1300)^{2}$, have been announced thanks

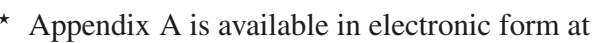
http://www . aanda.org

$\star \star$ Spectra are only available at the CDS via anonymous ftp to cdsarc.u-strasbg.fr (130.79.128.5) or via http://cdsarc.u-strasbg.fr/viz-bin/qcat?J/A+A/569/A120 $\star \star \star$ Based on observations collected at the European Organisation for Astronomical Research in the Southern Hemisphere, Chile.

$\star \star \star \star$ Based on observations made with the Gran Telescopio Canarias (GTC), installed in the Spanish Observatorio del Roque de los Muchachos of the Instituto de Astrofísica de Canarias, in the island of La Palma (program program GTC65-13B).

1 See the exoplanet.eu repositary for a complete list of planets and references.

2 The full list is available in DwarfArchives.org
}

to the advent of large-scale surveys such as the DEep Near Infrared Survey (DENIS; e.g. Epchtein et al. 1997; Delfosse et al. 1997; Martín et al. 1997, 1999), the Two Micron All Sky Survey (2MASS; e.g. Skrutskie et al. 2006; Burgasser et al. 1999, 2003b; Cruz et al. 2007), the Sloan Digital Sky Survey (SDSS; e.g. York et al. 2000; Leggett et al. 2000b; Geballe et al. 2002; Chiu et al. 2006), the UKIRT Infrared Deep Sky Survey (UKIDSS; Lawrence et al. 2007; Lodieu et al. 2007; Pinfield et al. 2008; Burningham et al. 2010, 2013), and the Wide-field Infrared Survey Explorer (WISE; Wright et al. 2010; Kirkpatrick et al. 2011; Mace et al. 2013; Thompson et al. 2013). However, only one wide brown dwarf companion co-moving with a planet-host star has been reported to date, HD 3651B (T7.5; $d=11.1 \pm 0.1 \mathrm{pc}$; Mugrauer et al. 2006b; Liu et al. 2007; Leggett et al. 2007; Burgasser 2007).

The presence of companion(s) to planet-host stars is thought to affect the efficiency of planet formation as well as the properties of any planet orbiting the primary star (Boss 2006; Nelson 2003; Moriwaki \& Nakagawa 2004) due to several processes, such as gravitational instability, accretion, velocities of colliding planetesimals, dissipation, and/or Kozai effects. Looking at a sample of close and wide companions to planet-host stars can reveal peculiarities in the eccentricity or mass distribution within those systems that may constrain current models of planet 
formation. In other words, one would like to address the following question: do companions to planet-host stars influence the properties of the planetary systems and to what degree?

Some planet-host stars are members of well-known multiple systems, such as 16 Cyg for example (Mason et al. 2001). Eggenberger et al. (2004) announced a sample of 15 new wide low-mass companions to planet-host stars and suggested a possible difference in the mass-period and eccentricity-period distributions, trend previously reported in Zucker \& Mazeh (2002). These discoveries and potential differences in the properties of planets orbiting single stars and planets orbiting stars in multiple systems triggered dedicated searches by several groups (Butler et al. 1997; Mugrauer et al. 2004a,b, 2005a,b, 2006a, 2007a,b; Konacki 2005; Eggenberger et al. 2006; Desidera \& Barbieri 2007; Bonavita \& Desidera 2007) to confirm (or otherwise) those trends in the properties of exoplanets. These searches also led to the discoveries of the first wide brown dwarf companion to HD 3651 (Mugrauer et al. 2006b), a K7 dwarf with a sub-Saturn mass planet (Fischer et al. 2004; Santos et al. 2005), and the most evolved multiple system with an exoplanet (Butler et al. 2001), composed of a K subgiant and a white dwarf (HD 27442; Chauvin et al. 2006; Raghavan et al. 2006; Mugrauer et al. 2007a). Moreover, several close companions have been announced around planet-host stars, thanks to highresolution adaptive optics imaging (Els et al. 2001; Patience et al. 2002) and lucky imaging (Ginski et al. 2012). All these surveys point towards a global multiplicity of at least $20 \%$ among solartype stars with exoplanets (Mugrauer et al. 2007a).

In this paper, we describe a new search for wide companions to planet-host stars taking advantage of the homogeneous coverage of the Visible and Infrared Survey Telescope for Astronomy (VISTA; Emerson 2001; Emerson et al. 2004b; Dalton et al. 2006) Hemisphere Survey (VHS; McMahon 2012) that will provide a full coverage of the southern sky in the upcoming years. We cross-correlated VHS with 2MASS (Cutri et al. 2003; Skrutskie et al. 2006) over an area of $~ 6200$ square degrees. Our work represents an extension and a complementary approach to the techniques employed by other authors (e.g. Mugrauer et al. 2007a). In Sect. 2 we present the characteristics of the 2MASS and VHS surveys as well as our astrometric and photometric search criteria. In Sect. 3 we describe our optical and near-infrared spectroscopic follow-up of companion candidates conducted with the spectrographs mounted on the New Technology Telescope (NTT) at La Silla Observatory in Chile and the OSIRIS instrument on the $10.4 \mathrm{~m}$ Gran Telescopio de Canarias at the Roque de Los Muchachos observatory on the island of La Palma (Canary Islands, Spain). In Sects. 4 and 5 we present the optical spectral classification scheme and the absolute magnitude vs spectral type relation employed throughout our analysis. In Sect. 6 we analyse the properties of the new wide companions found in our sample, including a brown dwarf binary candidate co-moving with HIP 70849. In Sects. 7-9 we discuss the stability of the widest companions in our sample, derive the frequency of planet-host stars at wide separations, and examine the dynamical properties of the systems with respect to previous studies (Eggenberger et al. 2004; Mugrauer et al. 2007a; Desidera \& Barbieri 2007).

\section{Surveys and search criteria}

\subsection{The $2 M A S S$ database}

The 2MASS project imaged the full sky in three near-infrared filters, $J(1.25 \mu \mathrm{m}), H(1.65 \mu \mathrm{m})$, and $K_{\mathrm{s}}(2.17 \mu \mathrm{m})$, down to $10 \sigma$ depths of $15.8 \mathrm{mag}, 15.1 \mathrm{mag}$, and $14.3 \mathrm{mag}$, respectively $(\text { Skrutskie et al. 2006) })^{3}$. The survey was conducted with $256 \times 256 \mathrm{HgCdTe}$ detectors installed on two automated $1.3-\mathrm{m}$ telescopes located on Mount Hopkins in Arizona (USA) and on Cerro Tololo (CTIO) in Chile. The observing strategy consisted of scanning the sky in three filters simultaneously with patches on the sky covering six degrees in declination and 8.5 arcmin in right ascension. The northern site started operations in 1997 , followed by the southern site a year later. The survey ended at the beginning of 2001, providing a final world-public catalogue of 471 million sources (Cutri et al. 2003). Comparison of the 2MASS catalogue with other catalogues such as Tycho2 (Høg et al. 2000) and UCAC (Zacharias et al. 2003) indicate that 2MASS positions in the International Celestial Reference System are accurate to 70-80 mas (root-mean-square; rms) over most of the magnitude range ( $K_{\mathrm{s}}$ between 6 and $\left.15 \mathrm{mag}\right)$. This all-sky survey played a major role in astronomy over the past decade, in particular in our knowledge of the low-mass and substellar populations of the solar neighbourhood (e.g. Reid et al. 1999; Burgasser et al. 1999; Burgasser 2004; Cruz et al. 2007; Kirkpatrick et al. 2010).

\subsection{The VISTA Hemisphere survey}

VISTA is a 4-m telescope (Emerson 2001; Emerson et al. 2004b) located at the Cerro Paranal Observatory (Chile) run by the European Southern Observatory (ESO). The telescope is equipped with the world's largest infrared camera, VIRCAM (Dalton et al. 2006) composed of 67 million pixels offering a field-of-view of 1.65 degrees in diameter, 0.6 square degrees of which is sampled by "pawprints" of 16 non-contiguous detectors with 0.34 arcsec pixels. Six suitably offsets and jittered pawprints are combined into a filled 1.5 square degree "tile", in which each piece of sky has been sampled by at least 2 pixels (ignoring the jitters), together with two additional $5.5 \times 88.5$ arcmin strips at opposite sides of the tile which are just covered once. The VISTA astrometric requirements, to an airmass of 2, are twofold: differential astrometric accuracies of 0.1 and 0.03 arcsec rms within the field covered by the full mosaic and each individual detector, respectively (Emerson et al. 2004a).

Seventy-five percent of the time available on VISTA is dedicated to ESO public surveys ${ }^{4}$, one of them being the VHS (McMahon 2012) planned to image the entire southern sky in $J$ and $K_{\mathrm{s}}$ over several years. Five thousands square degrees linked to the Dark Energy Survey (DES) ${ }^{5}$ will be observed in $J H K_{\mathrm{s}}$ whereas another 5000 square degrees around the Galactic caps will be imaged in $Y J H K_{\mathrm{s}}$. The averaged $5 \sigma$ depths measured for VHS are $J=19.5 \pm 0.3 \mathrm{mag}$ and $K_{\mathrm{s}}=18.5 \pm 0.3 \mathrm{mag}$. One of the main scientific drivers of the VHS includes the search for and characterisation of the nearest and coolest low-mass stars and brown dwarfs. The current VHS data release is DR $2^{6}$, made public on 17 October 2011 via the VISTA Science Archive (Hambly et al. 2008; Cross et al. 2012), but we also accessed data through flat-files made available to the VHS consortium.

\subsection{Search criteria}

We took advantage of the $>10$ year baseline between the VHS and 2MASS databases (McMahon 2012; Cutri et al. 2003; Skrutskie et al. 2006) to search for wide companions to

\footnotetext{
3 http://www.ipac.caltech.edu/2mass/

4 www.eso.org/sci/observing/PublicSurveys/

sciencePublicSurveys.html

5 http://www. darkenergysurvey.org/

6 http://www . eso.org/sci/observing/phase3/data_ releases/vhs_dr2.pdf
} 
Table 1. Wide common proper motion companions to planet-host stars.

\begin{tabular}{|c|c|c|c|c|c|c|c|c|c|c|c|c|c|c|}
\hline $\begin{array}{c}\text { RA } \\
\text { hh:mm:ss.ss }\end{array}$ & $\begin{array}{l}\text { Dec } \\
{ }^{\circ}:^{\prime \prime}\end{array}$ & $\begin{array}{c}J \\
\mathrm{mag}\end{array}$ & $\begin{array}{c}K_{\mathrm{s}} \\
\mathrm{mag}\end{array}$ & $\begin{array}{c}w 1 \\
\mathrm{mag}\end{array}$ & $\begin{array}{c}w 2 \\
\text { mag }\end{array}$ & $\begin{array}{r}\mu_{\alpha} \cos \delta \\
\operatorname{mas} / \mathrm{yr}\end{array}$ & $\begin{array}{c}\mu_{\delta} \\
\mathrm{mas} / \mathrm{yr}\end{array}$ & Name & $\begin{array}{l}\text { dist } \\
\text { pc }\end{array}$ & $\underset{\text { Sep }}{\operatorname{arcmin}}$ & $\begin{array}{c}\mu_{\alpha} \cos \delta \\
\text { mas/yr }\end{array}$ & $\begin{array}{c}\mu_{\delta} \\
\mathrm{mas} / \mathrm{yr}\end{array}$ & $\mathrm{SpT}$ & $\mathrm{C}$ ? \\
\hline $14: 26: 45.74$ & $-05: 10: 20.9$ & 12.116 & 11.279 & 11.060 & 10.921 & -140 & -145 & HD $126614(\mathrm{~K} 0 \mathrm{~V}+\mathrm{MV})$ & 72.4 & 0.70 & $-152.04 \pm 0.86$ & $-147.93 \pm 0.76$ & $\mathrm{M} 4.5 \pm 0.5$ & $\mathrm{Y}$ \\
\hline $14: 28: 42.32$ & $-46: 28: 41.7$ & 15.533 & 15.582 & 15.174 & 13.853 & -35 & -196 & HIP 70849 (K7Vk) & 24.0 & 6.29 & $-47.12 \pm 2.15$ & $-203.52 \pm 1.93$ & $\mathrm{~T} 4.5 \pm 0.5$ & $\mathrm{Y}$ \\
\hline $22: 31: 08.09$ & $-49: 26: 59.0$ & 12.415 & 11.595 & 11.208 & 11.030 & -143 & -191 & HD $213240(\mathrm{G} 0 / \mathrm{G} 1 \mathrm{~V})$ & 40.6 & 1.59 & $-136.01 \pm 0.48$ & $-194.44 \pm 0.33$ & M5-M5.5 & $\mathrm{Y}$ \\
\hline $11: 00: 49.99$ & $-11:$ & 14.062 & $\overline{42}$ & 13.034 & 12.816 & -179 & -20 & BD 10-3166 (K0V) & 66.0 & 8.86 & $-185.50 \pm 1.80$ & $-5.20 \pm 1.80$ & M5-M5.5 & Y: \\
\hline $12: 37: 18.37$ & $-39: 53: 14.8$ & 12.350 & 11.542 & 11.046 & 10.899 & -185 & -9 & HD 109749 (G3V) & 56.3 & 55.48 & $-157.85 \pm 0.93$ & $-5.43 \pm 0.72$ & $\mathrm{M} 3.5 \pm 0.5$ & Y: \\
\hline $15: 58: 41.31$ & $-44: 27: 54.2$ & 14.568 & 13.749 & 591 & 13.324 & -176 & -79 & HD 143361 (G6V) & 65.66 & 33.76 & $-158.32 \pm 1.37$ & $-118.21 \pm 1.01$ & M6-M6.5 & Y: \\
\hline 97 & $-26: 36: 42.6$ & 13.912 & .039 & 32 & 12.625 & 133 & -117 & HD 2 & 54.4 & 38.57 & \pm 0.97 & \pm 0.52 & $\mathrm{M} 6.5 \pm 0.5$ & Y: \\
\hline $23: 13$ & $-08: 22: 56.8$ & 12.036 & .236 & 016 & 10.838 & 361 & -23 & $91 \mathrm{Aqr}(\mathrm{KOIII})$ & 45.9 & 52.91 & $368.68 \pm 0.40$ & $-17.16 \pm 0.28$ & $\mathrm{M} 3.5 \pm 0.5$ & $\mathrm{Y}: ?$ \\
\hline $01: 48: 24.30$ & $-53: 33: 49.6$ & 16.332 & 15.635 & 15.523 & 15.670 & 133 & -91 & HD 10647 (F9V) & 17.3 & 53.63 & $166.32 \pm 0.24$ & $-106.52 \pm 0.27$ & - & $\mathrm{N}$ \\
\hline .82 & $-12: 4$ & 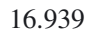 & .645 & 00 & 96 & -165 & 7 & V) & 55.0 & 39.20 & \pm 0.49 & .34 & - & $\mathrm{N}$ \\
\hline 12: & -40 : & 11. & 54 & 02 & 10.760 & -188 & -3 & 749 (G3V) & 56.3 & 39.19 & $-157.85 \pm 0.93$ & 0.72 & K7 & $\mathrm{N}$ \\
\hline 6.44 & $-45: 11: 05.2$ & 13.040 & 12.466 & 12.200 & 12.220 & -136 & -119 & HD 143361 (G6V) & 65.66 & 45.06 & $-158.32 \pm 1.37$ & $-118.21 \pm 1.01$ & Early & $\mathrm{N}$ \\
\hline & $-42: 35: 39.8$ & 15.5 & & & & -194 & -147 & HD 1 & 58.7 & 42.34 & \pm 1.04 & $-167.00 \pm 0.81$ & $\operatorname{sdM} 0.5 \pm 0.5$ & $\mathrm{~N}$ \\
\hline$: 02.53$ & $-42: 34: 54.8$ & 12.7 & 12.097 & & & -214 & -175 & HD 159868 (G5V) & 58.7 & 40.69 & $2 \pm 1.04$ & $-167.00 \pm 0.81$ & $\mathrm{M} 3.0 \pm 0.5$ & $\mathrm{~N}$ \\
\hline 23.80 & $-47: 5$ & & & & & 215 & -89 & HD 216435 (G0V) & 32.62 & 41.95 & $217.04 \pm 0.37$ & $-81.76 \pm 0.29$ & M4-M4.5 & $\mathrm{N}$ \\
\hline $23: 40: 52.27$ & $-05: 45: 01.4$ & 14.687 & 31 & 13.713 & 13.372 & -125 & -116 & HD 222582 (G5) & 42.0 & 20.41 & $-144.88 \pm 0.88$ & $-111.93 \pm 0.56$ & - & $\mathrm{N}$ \\
\hline
\end{tabular}

Notes. We list their coordinates (J2000), $J$ and $K_{\mathrm{s}}$ photometry from VHS, $w 1$ and $w 2$ magnitudes from WISE, proper motions in right ascension and declination (mas/yr) with a typical errors of 15-20 mas/yr in each direction, the name of the associated planet-host star with its spectral type in brackets, the distance of the planet-host star, the separation in arcmin, proper motions of the primary (in mas/yr) with their errors from HIPPARCOS, and the final conclusion (C?) on the validity of the system. From top to bottom, panels list objects with "Y" which are truly wide common proper motion systems, objects classified as "Y:" that are common proper motion candidates located at very wide separations, and rejected systems marked as "N".

planet-host stars imaged by the VHS. We searched for common proper motion companions to planet-host stars over a separation of 60 arcmin. We identified a total of 49 planet-host stars in the $\sim 6200$ square degrees (assuming 1.2 square degree coverage for the 5175 files) covered by VHS up to 31 October 2012. Among those 49 stars, 12 have incomplete coverage out to 60 arcmin at the time of writing, yielding a complete search for wide companions around 37 planet-host stars. Our search probes a similar parameter space as the hunt for wide binaries in the rNLTT catalogue (Gould \& Salim 2003; Salim \& Gould 2003) described by Chanamé \& Gould (2004), except that we focus here on planethost stars. We applied the following criteria to our photometric and astrometric search:

- sources brighter than $J_{\mathrm{VHS}}=17$ mag to ensure a reliable photometric detection in 2MASS;

- difference in $J$-band magnitude between 2MASS and VHS photometry less than 0.5 mag to remove unreliable photometry from 2MASS and VHS at the faint and bright ends, respectively;

- sources with separations larger than 1 arcsec between 2MASS and VHS;

- separations from the primary up to 60 arcmin;

- differences of less than 40 mas/yr (typically 2-3 $\sigma$ of the rms determined from stars in the fields-of-view of our targets) between each component of the proper motion of the candidate(s) and the planet-host star, corresponding to a maximum total difference of 56 mas/yr.

This query returned 16 wide co-moving proper motion candidates to 14 planet-host stars, including one brown dwarf candidate with the typical colours of mid-T dwarfs (Sect. 6.3). We list the coordinates $(\mathrm{J} 2000)$, near-infrared $\left(J, K_{\mathrm{s}}\right)$ photometry from VHS (McMahon 2012), mid-infrared $(w 1, w 2)$ photometry from the WISE all-sky release (Wright et al. 2010), proper motions in mas/yr for the primary and co-moving candidate from our VHS-WISE cross-match, physical separations, final spectral types (Sect. 6), and conclusions on the true companionship of the candidates in Table 1.

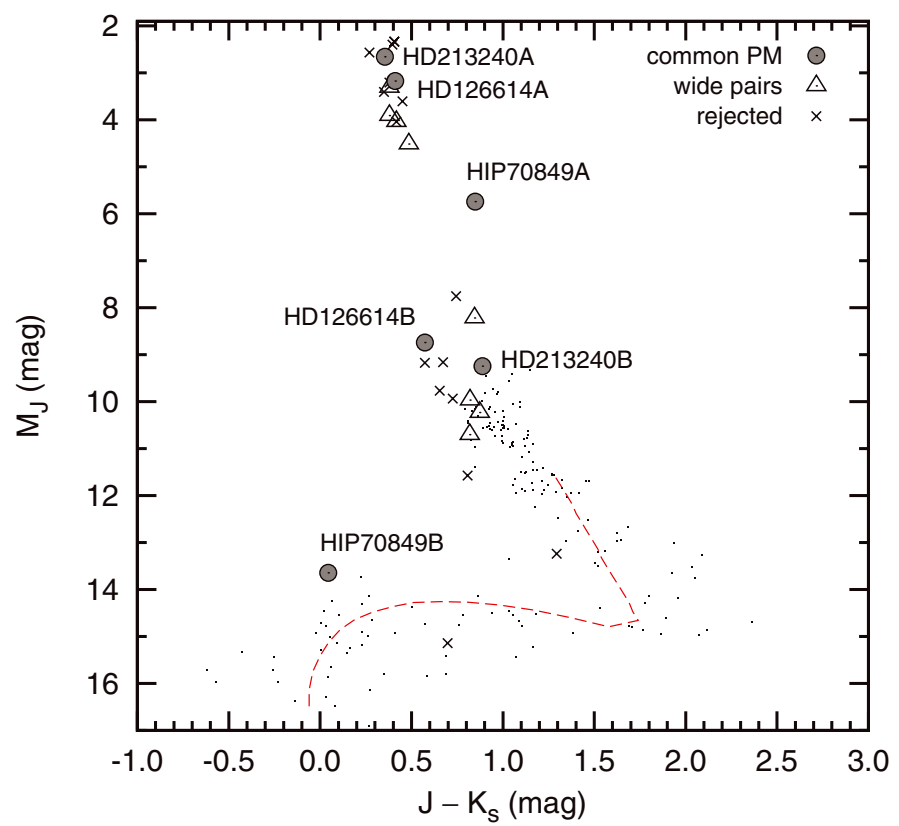

Fig. 1. $\left(J-K_{\mathrm{s}}, M_{J}\right)$ colour-absolute magnitude diagram for our wide co-moving companion candidates to planet-host stars surveyed by VHS and 2MASS. Filled circles indicate candidates confirmed spectroscopically as companions, open triangles indicate very wide co-moving pairs, whereas crosses mark rejected candidates.

We plot our candidates in the $\left(J-K_{\mathrm{s}}, M_{J}\right)$ colour-absolute magnitude diagram displayed in Fig. 1, where we assumed the planet-host star distance to determine a possible absolute $J$-band magnitude. We note that the companion to HD 213240 was already published by Mugrauer et al. (2005b) and that Desidera \& Barbieri (2007) already reported a wide companion to HD 109749 but at a closer separation.

We were able to discard three candidates photometrically before carrying out the spectroscopic follow-up i.e. both components of the system don't seem to be located at the same distance 
in the colour-absolute magnitude diagram (e.g. Fig. 1). We found these candidates around HD 10647, HD 103774 (also discarded astrometrically after inspection of the images), and HD 222582 (Table 1) but we do not discuss them further below.

We estimate the typical dispersion observed in the vector point diagram for the sources identified in 2MASS and VHS to be of the order of 15-20 mas. In the case of three co-moving candidates to planet-host stars discussed at length in Sect. 6, we derive dispersions along both axes of approximately 15-20 mas/yr Moreover, we should take into account possible orbital motions, which we estimate to be less than 9-12 mas over 12 years (typical baseline between 2MASS and VHS). For our calculation, we considered again the case of the three confirmed companions (see Sect. 6). Their spectroscopic distances lie in the range 24-72 pc and their physical separations are larger than $3000 \mathrm{au}$. Moreover, we assumed longitudinal motions (the worst case) with an amplitude of twice the separation over half of the period of the binary systems. Hence, we conclude that orbital motion is negligible because its effect is below the uncertainty on our proper motion determination. We note that Chanamé \& Gould (2004) found a general excess of proper motion differences for separations larger than 42 arcsec but for closer systems.

\subsection{Known planet-host star binaries in VHS}

We checked how many of the known planet-host stars within the VHS area considered in this work are known multiple systems in the literature to gauge the completeness of our search. We identified a total of six wide or relatively wide systems which we briefly describe below.

1. HD 109749 is a double G3IV star at 59 pc (van Leeuwen 2007) with a physical separation of approximately $750 \mathrm{au}$. The system harbours a $0.28 M_{\text {Jup }}$ planet with a period of 5.24 days (Nordström et al. 2004; Fischer et al. 2006).

2. HD 126614 is a metal-rich $(\mathrm{Fe} / \mathrm{H}=+0.56 \mathrm{dex}) \mathrm{K} 0$ star resolved in two components separated by 0.5 arcsec, corresponding to a projected physical separation of $\sim 36$ au at a distance of $72.4 \pm 5.4 \mathrm{pc}$ (Howard et al. 2010). A second M dwarf, NLTT, 37349, has been reported as a wide companion by Gould \& Chanamé (2004).

3. HR 6 (= HD 142) is a double star at 20.6 pc (van Leeuwen 2007) whose components are separated by approximately 85 au (Fabricius et al. 2002; Gray et al. 2006). The star is orbited by three planets announced by Tinney et al. (2002) and Wittenmyer et al. (2012).

4. HR 8734 (= HD 217107) is a G8IV double star at $19.72 \mathrm{pc}$ (Montes et al. 2001; van Leeuwen 2007) made of the CDM J22583-0224A and CDM J22583-0224B components (Høg et al. 2000; Fabricius et al. 2002) with a separation of $\sim 10$ au and orbited by two Jupiter-mass planets (Wright et al. 2009).

5. HD 28254 is a metal-rich $(\mathrm{Fe} / \mathrm{H}=+0.36 \mathrm{dex}) \mathrm{G} 1 \mathrm{IV} / \mathrm{V}$ star at $56.2 \mathrm{pc}$ (van Leeuwen 2007). It includes the components CCDM J04248-5037A $(J=6.395 \mathrm{mag})$ and CCDM J04248-5037B ( $J=7.60 \mathrm{mag})$ separated by $\sim 240$ au and a $1.16 M_{\text {Jup }}$ planet with a long period ( $P=1116$ days; Naef et al. 2010).

6. Gl 86 is a metal-poor G9V star (Torres et al. 2006) at $10.9 \mathrm{pc}$ (van Leeuwen 2007) with a white dwarf companion (G1 86B; Els et al. 2001; Mugrauer \& Neuhäuser 2005; Lagrange et al. 2006) at a separation of $\sim 17.4 \mathrm{au}$. Queloz et al. (2000) announced a $4 M_{\text {Jup }}$ planet orbiting Gl 86 in $\sim 15.8$ days.

We do not recover any of these planet-host stars because each component is very bright $\left(J_{2 \mathrm{MASS}}=4.7-8.8 \mathrm{mag}\right)$, leading to
Table 2. Log of the spectroscopic observations.

\begin{tabular}{ccccc}
\hline \hline $\begin{array}{c}\text { RA } \\
\text { hh:mm:ss.ss }\end{array}$ & $\begin{array}{c}\text { Dec } \\
\text { o }^{\prime \prime}\end{array}$ & Tel. Inst. & $\begin{array}{c}\text { Date } \\
\text { dd/mm/yy }\end{array}$ & $\begin{array}{c}\text { ExpT } \\
\mathrm{s}\end{array}$ \\
\hline $01: 48: 24.30$ & $-53: 33: 49.6$ & no spectrum & - & - \\
11:00:49.99 & $-11: 03: 43.2$ & GTC OSIRIS & $04 / 11 / 13$ & 240 \\
11:57:01.82 & $-12: 45: 39.0$ & no spectrum & - & - \\
12:37:18.37 & $-39: 53: 14.8$ & NTT EFOSC2 & $11 / 03 / 14$ & $4 \times 300$ \\
12:40:36.35 & $-40: 59: 00.7$ & NTT EFOSC2 & $11 / 08 / 13$ & 300 \\
14:26:45.74 & $-05: 10: 20.9$ & NTT EFOSC2 & $11 / 03 / 14$ & $3 \times 600$ \\
14:28:42.31 & $-46: 28: 41.7$ & NTT SofI & $29 / 12 / 12$ & $960+1200$ \\
15:58:41.31 & $-44: 27: 54.2$ & NTT EFOSC2 & $11 / 08 / 13$ & 1800 \\
16:01:36.44 & $-45: 11: 05.2$ & NTT EFOSC2 & $11 / 03 / 14$ & $3 \times 600$ \\
17:36:35.14 & $-42: 35: 39.8$ & NTT EFOSC2 & $11 / 08 / 13$ & 1200 \\
17:41:02.53 & $-42: 34: 54.8$ & NTT EFOSC2 & $11 / 08 / 13$ & 600 \\
21:51:24.97 & $-26: 36: 42.6$ & NTT EFOSC2 & $11 / 08 / 13$ & 1800 \\
22:31:08.09 & $-49: 26: 59.0$ & NTT EFOSC2 & $11 / 08 / 13$ & 600 \\
22:53:23.80 & $-47: 54: 01.8$ & NTT EFOSC2 & $11 / 08 / 13$ & 600 \\
23:13:45.29 & $-08: 22: 56.8$ & NTT EFOSC2 & $11 / 08 / 13$ & 600 \\
23:40:52.27 & $-05: 45: 01.4$ & no spectrum & - & - \\
\hline
\end{tabular}

Notes. We give two integration times for the SofI observations because we observed the object with the blue and red gratings to cover the full 0.9 to 2.5 micron wavelngth range.

differences in the $J$-band magnitudes between 2MASS and VHS larger than $0.5 \mathrm{mag}$, our upper limit (Sect. 2.3). This effect comes from the heavy saturation effects seen for these stars on the VHS images. Furhermore, HR 8734 has a very low proper motion in both directions whereas the mean proper motions of HD 109749 and HD 28254 are slightly larger than 0.15 arcsec/yr. Moreover, HD 126614 is unresolved on the seeing-limited VHS images.

\section{Spectroscopic follow-up of potential companions}

\subsection{NTT/EFOSC2 optical spectroscopy}

We obtained optical spectroscopy of eight of the ten new systems with the ESO Faint Object Spectrograph and Camera (EFOSC2; Buzzoni et al. 1984) installed on the 3.58-m NTT in La Silla (Chile) on 11 August 2013 in visitor mode (Table 2). The observations of our candidates took place between $\mathrm{UT}=0 \mathrm{~h}$ and $5 \mathrm{~h}$. The night was non photometric with thin cirrus all night. The moon was illuminated at $48 \%$ and was up until UT $=5 \mathrm{~h}$. The seeing oscillated between 1 and 1.7 arcsec during that night. We observed three other candidates with the same set-up on 11 March 2014, a clear night with photometric conditions and seeing between 0.7 and 0.9 arcsec. We observed our targets at the end of the night when the moon had set.

EFOSC2 is equipped with a Loral/Lesser, thinned, ultraviolet flooded $2048 \times 2048$ chip sensitive to optical wavelengths. The $2 \times 2$ binning mode offers a pixel scale of 0.24 arcsec and a field-of-view of $4.1 \times 4.1$ arcmin. We used grism \#5 with a slit of 1 arcsec for spectroscopic observations, covering the 520$935 \mathrm{~nm}$ wavelength range with a resolution of $1.54 \mathrm{~nm}$. We obtained single or multiple on-source integrations between $300 \mathrm{~s}$ and $1800 \mathrm{~s}$ at parallactic angle, depending on the brightness of the object (Table 2). We observed internal flat fields straight after the spectra of the targets to correct for fringing at red wavelengths. We also obtained bias and Helium-Argon arc frames during the afternoon preceding our observations as well as one spectro-photometric standard star per run observed in between our targets to calibrate them in flux (LTT7379 (G0); HR 5501 


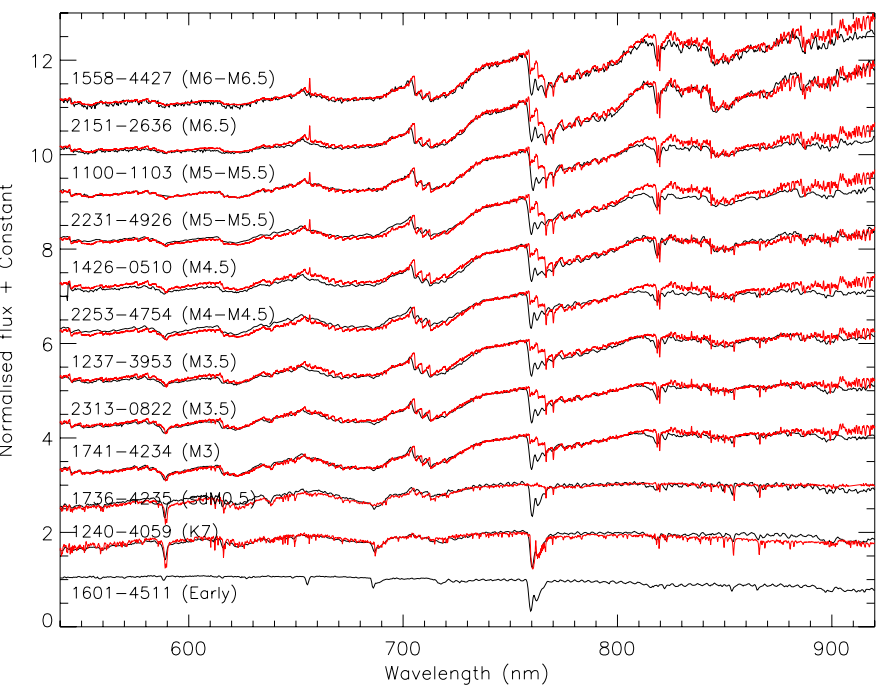

Fig. 2. Low-resolution optical spectra for wide companion candidates obtained with NTT/EFOSC2 and GTC/OSIRIS (Table 2). The names of the candidate companions and final spectral types are written on top of each spectrum. Spectra are ordered by increasing spectral types.

(B9.5) van Altena et al. 1995; Gontcharov 2006; van Leeuwen 2007; Pancino et al. 2012) .

We carried out the data reduction of all optical spectra under the IRAF environment (Tody 1986, 1993) ${ }^{7}$. We subtracted the raw median-combined bias frame from the raw spectrum and divided by a normalised internal flat field obtained at the same position of our targets to minimize the fringing in the red part of the spectrum. We extracted a one-dimensional (1D) spectrum, choosing an optimal sky background and aperture. In the case of multiple exposures, we combined the spectra before conducting the extraction. We calibrated our spectrum in wavelength with Helium-Argon arc lamps before calibrating it in flux, using the spectro-photometric standard star. The final optical spectra of our targets, normalised at $750 \mathrm{~nm}$, are displayed in Fig. 2.

\subsection{GTC/OSIRIS optical spectroscopy}

We obtained low-resolution $(R=500)$ optical spectroscopy with the OSIRIS (Optical System for Imaging and lowintermediate Resolution Integrated Spectroscopy; Cepa et al. 2000) mounted on the 10.4-m GTC telescope in La Palma. We observed the potential companion to BD 10-3166 on 4 November 2013 in service mode (Table 2) as part of program GTC65-13B (PI Lodieu).

OSIRIS is equipped with two $2048 \times 4096$ Marconi CCD4282 detectors offering a field-of-view approximately $7 \times 7$ arcmin with an unbinned pixel scale of 0.125 arcsec. The night was dark with spectroscopic conditions and a seeing of 1.7 arcsec. We used a slit of 1.0 arcsec, the R500R grating and a single onsource integration of $240 \mathrm{~s}$. Bias frames, dome flat fields, and Neon, Xenon, and $\mathrm{HgAr}$ arc lamps were observed by the observatory staff during the afternoon preceding the observations.

We reduced the OSIRIS optical spectrum in a very similar way as the EFOSC2 spectra. We substracted the raw spectrum by a median-combined bias and divided by a normalised

\footnotetext{
7 IRAF is distributed by the National Optical Astronomy Observatories, which are operated by the Association of Universities for Research in Astronomy, Inc., under cooperative agreement with the National Science Foundation.
}

median-combined dome flat field taken during the afternoon. We extracted optimally a 1D spectrum from the 2D image and calibrated that spectrum in wavelength with the lines from the combined arc lamp. We calibrated our spectrum in flux with the GD 248 spectro-photometric standard (Farihi et al. 2005; Landolt \& Uomoto 2007) corrected for the second-order contamination. We corrected the second-order by joining the response of the detector from the observations of the standard star with the R500R grating and the same grating in combination with the Sloan $z$ broad-band filter. The final GTC spectrum of 2MASS 11004999-1103432, normalised at $750 \mathrm{~nm}$, is displayed in Fig. 2.

\subsection{NTT/Sofl near-infrared spectroscopy}

We performed near-infrared spectroscopy of the wide companion to HIP 70849 on 29 December 2012 with the Son of ISAAC (SofI) instrument mounted on the 3.5-m NTT (Moorwood et al. 1998) in La Silla, Chile (Table 2). SofI is equipped with a Hawaii $\mathrm{HgCdTe} 1024 \times 1024$ array with squared 18.5 micron pixels and offers imaging and spectroscopic capabilities. The pixel scale is 0.292 arcsec in the Large Field configuration, offering a $4.9 \times 4.9$ arcmin field-of-view. We observed our target with the blue $Z J$ and red $H K$ gratings, covering 0.9-1.64 and 1.53-2.52 microns, respectively, at a resolution of $\sim 600$ with a slit of 1 arcsec. We set one and two ABBA patterns with individual on-source integrations to $60 \mathrm{~s}$ repeated four and five times in the $Z J$ and $H K$ gratings, respectively, to correct for the sky. The total integration times are $20 \mathrm{~min}$ and $40 \mathrm{~min}$ for the blue and red gratings, respectively. We observed a telluric standard immediately after our target at a similar airmass to correct for telluric absorption, HIP 68930 (A0V; Houk 1978; van Leeuwen 2007). Dome flats and halogen arc lamps were taken during the afternoon preceding the beginning of the night with the same set-up as our observations.

Each individual frame was flat-fielded and sky-subtracted before a one-dimensional spectrum was extracted. We combined the individual one-dimensional spectra to increase the signal-tonoise. Then, we divided this combined spectrum of the target by the extracted spectrum of the standard star and later multiplied by an appropriate spectral template of spectral type A0 (containing the main hydrogen lines in absorption) smoothed to our resolution. The final one-dimensional spectrum of HIP 70849B, normalised at 1.25 microns, is shown in Fig. 3 along with known $\mathrm{T}$ dwarf spectral standards taken from the IRTF/SpeX library ${ }^{8}$.

\section{Optical spectral classification}

To classify the components of our potential wide multiple systems, we opted for the direct comparison of our optical spectra with known $\mathrm{M}$ dwarf templates created from the Sloan Digital Sky Survey (SDSS; York et al. 2000) spectroscopic database by Bochanski et al. (2007). This database contains a repository of good-quality M0-M9 spectra spanning the 380$940 \mathrm{~nm}$ wavelength range at a resolution of 2000. All spectra are wavelength- and flux-calibrated and corrected for telluric absorption. M dwarfs are classified based on the Hammer classification scheme (Covey et al. 2007) which uses the spectral energy distribution of stars over the $0.3-2.5$ micron range using photometry from SDSS and 2MASS. We added sub-type to this list of

\footnotetext{
8 http://pono.ucsd.edu/\$ $\backslash$ sim\$adam/browndwarfs/ spexprism/
} 


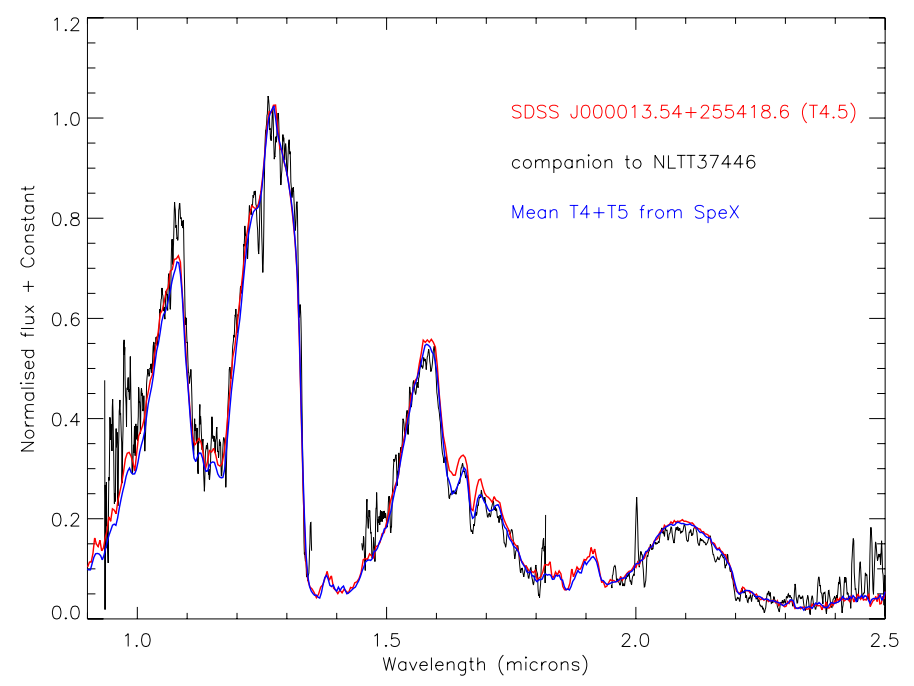

Fig. 3. NTT/SofI low-resolution near-infrared spectra of the T4.5 dwarf co-moving with HIP 70849 (black line). Overplotted are IRTF/SpeX spectra of SDSS J000013.54+255418.6 (T4.5; Knapp et al. 2004; Jameson et al. 2008; Burgasser et al. 2006) and the average spectrum of a T4 and T5 dwarf.

templates to provide a complete list of spectral templates with optical spectral types accurate to one sub-type.

In addition to this list of solar-metallicity $\mathrm{M}$ dwarf templates, we dowloaded the spectra of the brightest object of each sub-class of low-metallicty $M$ dwarfs, classified either as subdwarfs, extreme subdwarfs, or ultrasubdwarfs. The lowmetallicity classification follow the scheme by Lépine et al. (2007), which is an updated version of the original scheme proposed by Gizis (1997). For more details, we refer the reader to Espinoza Contreras et al. (2014).

We compared each of our optical spectra to this grid of spectral templates and assign a spectral type to the nearest half subclass, which we consider as our (absolute) uncertainty. All our targets are either solar-type M dwarfs or M subdwarfs, except 2MASS J12403635-4059007 which we classify as a K7 dwarf (Table 1).

\section{Absolute magnitude vs. spectral type relation}

Throughout this section and the rest of the paper, we will make use of the absolute magnitude vs. spectral type relations given in Table 3 to derive spectroscopic distances for the co-moving companion candidates to planet-host stars. We calculated the absolute $J$-, $H$-, and $K_{\mathrm{s}}$-band magnitudes using the 8 pc census of M dwarfs listed in Table 4 of Kirkpatrick et al. (2012). We compiled all M dwarfs with spectral types between M0 and M7 with parallaxes and accurate spectral types. We computed the average values (Table 3), after excluding some outliers and members of multiple systems with unresolved 2MASS photometry. We also list in brackets the dispersion in absolute magnitudes taken from the full sample of objects considered for each sub-type.

\section{New wide companions to planet-host stars}

In this section, we only describe the properties of the three planet-host stars with most probable wide companions and derive their main properties (Table 1) thanks to our spectroscopic follow-up (Sect. 3). The other companions, located at very wide separations or rejected as co-moving companions to planet-host stars are discussed in the Appendix A.
Table 3. Absolute $J, H$, and $K_{\mathrm{s}}$ magnitudes as a function of spectral types for M dwarfs with spectral types between M0 and M7 drawn from the census of 8 pc compiled by Kirkpatrick et al. (2012).

\begin{tabular}{lcccc}
\hline \hline SpType & $\begin{array}{c}M_{J} \\
\mathrm{mag}(\mathrm{err})\end{array}$ & $\begin{array}{c}M_{H} \\
\mathrm{mag}\end{array}$ & $\begin{array}{c}M_{K_{\mathrm{s}}} \\
\mathrm{mag}\end{array}$ & $\mathrm{Nb}$ \\
\hline M0.0 & $6.273(0.322)$ & $5.577(0.440)$ & $5.408(0.337)$ & 3 \\
M0.5 & $6.761(0.258)$ & $6.031(0.230)$ & $5.888(0.200)$ & 1 \\
M1.0 & $6.393(0.089)$ & $5.736(0.144)$ & $5.491(0.125)$ & 2 \\
M1.5 & $6.943(0.540)$ & $6.350(0.572)$ & $6.063(0.537)$ & 8 \\
M2.0 & $7.134(0.584)$ & $6.554(0.608)$ & $6.253(0.597)$ & 4 \\
M2.5 & $7.255(0.288)$ & $6.667(0.297)$ & $6.409(0.294)$ & 5 \\
M3.0 & $7.758(0.650)$ & $7.206(0.664)$ & $6.936(0.645)$ & 9 \\
M3.5 & $8.025(0.458)$ & $7.460(0.454)$ & $7.183(0.464)$ & 14 \\
M4.0 & $8.558(0.518)$ & $8.027(0.567)$ & $7.721(0.563)$ & 10 \\
M4.5 & $9.038(0.420)$ & $8.495(0.440)$ & $8.183(0.438)$ & 7 \\
M5.0 & $9.617(0.121)$ & $9.029(0.164)$ & $8.685(0.136)$ & 3 \\
M5.5 & $9.671(0.392)$ & $9.101(0.397)$ & $8.745(0.394)$ & 7 \\
M6.0 & $10.439(0.025)$ & $9.883(0.069)$ & $9.535(0.120)$ & 2 \\
M6.5 & $10.505(0.067)$ & $9.899(0.079)$ & $9.552(0.089)$ & 2 \\
M7.0 & $10.776(0.026)$ & $10.126(0.022)$ & $9.739(0.027)$ & 1 \\
\hline
\end{tabular}

Notes. The last column indicates the number of stars used to derive the average absolute magnitudes.

\subsection{HD 126614}

Howard et al. (2010) announced a $0.38 M_{\text {Jup }}$ planet with a period of 1244 days orbiting the metal-rich $(\mathrm{Fe} / \mathrm{H}=+0.56 \mathrm{dex})$ K0 star, HD 126614. These authors also resolved HD 126614 in two components separated by 0.5 arcsec (Howard et al. 2010), corresponding to a projected physical separation of $\sim 36$ au at a distance of $72.4 \pm 5.4 \mathrm{pc}$ (van Leeuwen 2007). However, if we apply a spectral type vs. absolute magnitude relation for K0 main-sequence stars using Gl 68 as a K0 template at 7.53 pc $(J=3.83$ mag; Gray et al. 2006; Kirkpatrick et al. 2012), we derive a spectroscopic distance of $\sim 40$ pc for HD 126614. This discrepancy might arise from two facts. On the one hand, HD 126614 is not a main-sequence K0 star but rather a metalrich K0 sub-giant because of its temperature (5541-5645 K) and mass (1.03-1.25 $M_{\odot}$; Howard et al. 2010; Santos et al. 2013). On the other hand, HD 126614 is a metal-rich star, with $[\mathrm{Fe} / \mathrm{H}]=0.46-0.60$ (Howard et al. 2010; Santos et al. 2013). Hence, the above relationship for solar-type K0 main-sequence star is not applicable so we favour the HIPPARCos distance.

We identified a relatively close companion at 41.8 arcsec from the planet-host system, previously reported by Gould \& Chanamé (2004) as being NLTT 37349. We confirm the common proper motion of the pair after examination of the optical and infrared images of the system taken by the Digital Sky Survey, 2MASS, and VHS over a period of more than 50 years. The common motion of the two sources over such long period adds strong credibility to their association.

We obtained an optical spectrum of this close companion and classify it as M4.5 \pm 0.5 dwarf. If a true companion, this M4.5 dwarf represents a very interesting $M$ dwarf with supersolar metallicity that is amenable for further investigation. We derive a spectroscopic distance of $41.3 \mathrm{pc}$ with a possible range of 29-65 pc, assuming that the absolute magnitude vs. spectral type relation is valid for (single) solar-metallicity $\mathrm{M}$ dwarfs. We note that, according to the Padova tracks (Bertelli et al. 2008), a $0.16 M_{\odot}$ dwarf is less luminous $(\sim 0.07 \mathrm{dex})$, cooler $(\sim 200 \mathrm{~K})$, and fainter by approximatly $0.1 \mathrm{mag}$ in the near-infrared than a solar-metallicity object with the same mass. Moreover, if this 
companion is an equal-mass binary, it would lie at a distance greater by $40 \%$ (41-91 pc). This value is in agreement with the spectroscopic distance of the primary and the range of possible distances is within $1.5 \sigma$ of the HIPPARCos distance. Hence, we argue that these two objects form a physical pair with a projected physical separation of approximately 3000 au (assuming a distance of $72.4 \mathrm{pc}$ ).

\section{2. $H D 213240$}

Santos et al. (2001) reported an eccentric $(0.45 \pm 0.05)$ planet with a mass of $4.5 M_{\text {Jup }}$ and a period of $\sim 950$ days, orbiting HD 213240, an old (5.11 Gyr) G0/G1V star (Houk 1978) at $40.6 \pm 1.0 \mathrm{pc}$ van Leeuwen (2007) and with a metallicity of $[\mathrm{Fe} / \mathrm{H}]=0.16$ (Nordström et al. 2004).

We unveiled a potential wide companion to HD 213240, located at 1.6 arcmin only, the closest companion found among our candidates. We classify this companion as a M5-M5.5 dwarf, translating into a spectroscopic distance of 29-42 pc.

We conclude that these two objects form a co-moving pair at a projected physical separation of $\sim 3900$ au, adopting the distance of the primary.

\subsection{A new brown dwarf companion to HIP 70849}

Ségransan et al. (2011) reported a $9 \pm 6 M_{\text {Jup }}$ planet orbiting HIP 70849 in a very eccentric orbit $(0.60 \pm 0.13)$ associated to a long period of $\sim 10000$ days. The planet-host star is a low-mass $\operatorname{star}\left(0.63 \pm 0.03 M_{\odot}\right)$ of spectral type K7V located $24.0 \pm 1.2 \mathrm{pc}$ (van Leeuwen 2007) from the Sun, an age of 1-5 Gyr, and a metallicity possibly close to solar.

We detected a potential wide proper motion companion at 6.3 arcmin from HIP 70849. We unambiguously confirm the association of the potential brown dwarf companion and the planet-host in the vector point diagram and in the $\left(J-K, M_{J}\right)$ colour-absolute magnitude diagram displayed in Fig. 4. We obtained near-infrared spectroscopy with NTT/SofI (Sect. 3.3), classifying the companion as a T4.5 dwarf (Fig. 3), hence, confirming its substellar status. It is the second brown dwarf directly imaged around a planet-host star after HD 3651 (Mugrauer et al. 2006b) and possibly the first brown dwarf binary at wide separation from a star orbited by a $9 M_{\text {Jup }}$ planet.

To check whether the binary is physically bound or a chance alignment, we have estimated the probability that the companion is a field T dwarf contaminant in our search. First, we have estimated that the expected number of T dwarfs in the full area of our search $\left(\sim 150 \mathrm{deg}^{2}\right.$ around the full sample of 49 stars $)$ is $\sim 1.3$. We have adopted a density of $5 \times 10^{-3}$ objects $/ \mathrm{pc}^{-3}$ (Burgasser et al. 2003a) and an average absolute magnitude of $M_{J} \sim 15 \mathrm{mag}$ (Vrba et al. 2004) for such objects, and considered that our search is limited to $J=17$ mag. Secondly, we have estimated the probability that one of this object has a proper motion within $40 \mathrm{mas} / \mathrm{yr}$ of the primary's proper motion in each direction. We have determined that about $20 \%$ of the high proper motion objects in our full catalogue have such similar proper motion as HD 70849. Hence, we estimate that the final probability that a field $\mathrm{T}$ dwarf lies at separations less than 60 arcmin of one of our primaries is of 0.27 . If we take into account that the T4.5 was found at a separation of 6.29 arcmin from the primary, the probability of contamination decrease by a factor of 100 , yielding a probability of 0.027 . These estimates combined with the similar spectroscopic distances derived for both components support that the T4.5 is a true physical companion of the HD 70849 planet-host star.

\section{Stability of wide companions}

Three of our planet-host star companions (HIP 70849B, HD 213240 B, HIP 126614 B) are located at angular separations between 0.7 and 6.3 arcmin, corresponding to physical separations in the 3000-9100 au range. The mass of their primaries lie between 0.63 and $1.22 M_{\odot}$. To study the dynamical stability of these systems, we can investigate their separations versus total masses (see Fig. 16 in Close et al. 2007). From this kind of diagrams, we can see that these three systems are located in the region that is dynamically stable for the typical stellar densities of the Solar vicinity.

However, the other planet-host star companions are located at physical separations of 100000-150000 au (0.50-0.75 pc). Given that the mass of their primaries are about $1 M_{\odot}$, these extremely wide binary systems are not dynamically stable at the typical density of field stars and the age of the Solar vicinity but do exist (Scholz et al. 2008; Shaya \& Olling 2011). Although binaries with separations larger than $10000 \mathrm{au}$ and up to 200,000 au have been reported in the literature (e.g. Makarov et al. 2008; Quinn \& Smith 2009; Poveda et al. 2009; Raghavan et al. 2010; Deacon et al. 2012), most of these systems are either young or expected to be disrupted at older ages or are halo binaries. Another possibility is that these systems could be disrupted binaries that drift apart quite slowly because disruption does not necessarily denote a violent phenomenon, suggesting that these pairs would survive as common proper motion associations for much longer than their disruption time-scales (Shaya \& Olling 2011). Following the analytical solutions given in Weinberg et al. (1987) and Binney \& Tremaine (1987) we estimate the timescale for disruption of these systems in an environment with typical densities of the solar vicinity ( $\sim 0.07 \mathrm{ob}-$ ject $\mathrm{pc}^{-3}$ ) of a several hundred million years. This is much lower than the expected age of these systems, considering that we have not found any evidence of youth in the spectra of the secondaries. These estimations suggest that these systems are not physically bound. Given the large uncertainties in the determination of their distances, they are either objects with similar proper motions that lie along the same line of sight by chance, but lie at different distances, or they are systems located at the same distance and share similar projected velocities. This may be the result of a common origin or a similar dynamical evolution, in the same way as stars which are members to moving groups.

We have estimated the probability of these five wide companions to be truly associated with the planet-host stars. We counted the numbers of high proper motion stars in the full 2MASS-VHS catalogue with similar motions as the planet-host star. We found 19553 objects that fulfill those criteria, implying that we would expect $9.9 \pm 3.1$ contaminants (assuming Poisson errors) around the 49 planet-host stars located in 6200 square degrees surveyed by the VHS (Table 1). However, this number represents an upper limit because we have added a distance criterion to our sample, based on our spectroscopic follow-up. We rejected eight of our candidates, in agreement with the minimum of seven contaminants. Additionally, we have five very wide companions sharing the same proper motion and distance as the planet-host stars. We conclude that among the 13 doubtful candidate companions in Table 1 a few could be part of comoving groups. We have three very wide companions with spectral types later than M5, whose number of field objects is typically lower than earlier spectral types, suggesting that those are most likely to be associated to th planet-host star (if any of these are). This result is not so surprising and in line with the conclusions of Chanamé \& Gould (2004) who argue that binaries with 
A\&A 569, A120 (2014)
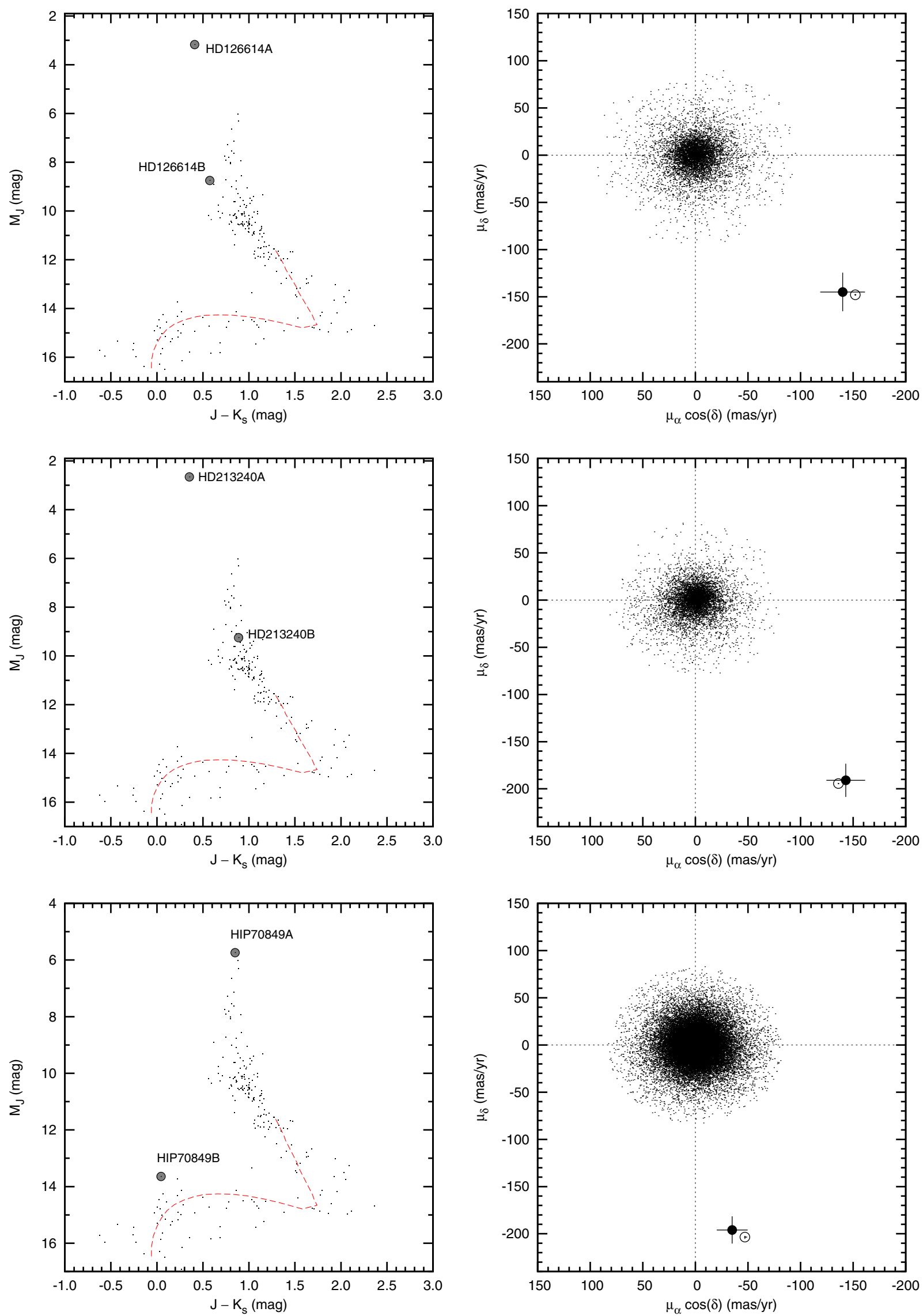

Fig. 4. Left: proper motion diagram for all point sources within 60 arcmin from the primary stars with confirmed wide common proper motion companions: HD 126614 (top), HD 213240 (middle), HIP 70849 (bottom). Right: colour-magnitude diagrams showing the position of the three planet-host stars and their companions. Overplotted are known M, L and T dwarfs with parallaxes from Leggett et al. (2000a), Vrba et al. (2004), and Kirkpatrick et al. (2012). 
separations larger than 8.3 arcmin belonging to the thin disk can not be identified reliably.

\section{Multiplicity of planet-host stars}

We have cross-correlated approximately 6200 square degrees of the southern sky imaged by VHS and 2MASS. We found 37 planet-host stars $(20 \mathrm{G}, 7 \mathrm{~F}, 10 \mathrm{~K}, 5 \mathrm{M})$ with a full VHS covereage within a radius of 60 arcmin and separations larger than 2 arcsec between 2MASS and VHS. In this complete sample, we confirmed spectroscopically one substellar companion, two M dwarf companions, and another four (or five if we add the companion to 91 Aqr) at separations larger than $100000 \mathrm{au}$. If we consider only dynamically-stable systems (see Sect. 7), we derive a frequency of wide ( $>50-150 \mathrm{au})$ stellar and substellar companions to planet-host stars of $2 / 37=5.4 \pm 3.8 \%$ and $1 / 37=2.7 \pm 2.7 \%$ ( $1 \sigma$ confidence level with poissonian errors $)$, respectively. Note that we have excluded from this estimate the known companions described in Sect. 2.4 because we do not recover them in our study (only two have projected separations larger than our lower limit). In any case, our multiplicity estimates represent lower limits to the true binary frequency of planet-host stars because our study is not sensitive to close systems (e.g. HD 126614 (K0+M); Howard et al. 2010).

Overall, our binary frequencies of a few percents are in agreement with the number of wide substellar companions to young solar analogues (0.5-6.3\% for separations in the 281590 au range; Metchev \& Hillenbrand 2009), M dwarfs (0.1$2.2 \%$ for separations larger than $\sim 120$ au ; Radigan et al. 2008), the frequency of $1 \pm 1 \%$ of brown dwarf companions to GKM stars in the 75-300 au range (McCarthy \& Zuckerman 2004) or the lack of planetary-mass objects more massive than 5-10 $M_{\text {Jup }}$ around young nearby stars beyond 36-65 au (Masciadri et al. 2005). Similarly, Raghavan et al. (2010) derived a stellar multiplicity of $7.3 \%$ and $2.2 \%$ among solar-type stars within $25 \mathrm{pc}$ for projected separations in the 1000-10000 au and 1000050000 au ranges, respectively (their Fig. 11 and Table 5). These estimates are in good agreement with our results. If we apply our photometric and astrometric selection constraints to the 454 solar-type stars listed in Raghavan et al. (2010), we found 11 wide common proper motion candidates to 74 stars $(21 \mathrm{~F}$, $33 \mathrm{G}, 20 \mathrm{~K}$ ) imaged by the VHS at the time of writing, corresponding to $11 / 74=13.5 \pm 4.0 \%$ if all are true companions. Only one candidate lies close to its G-type primary whereas the others lie at projected physical separations larger than $10000 \mathrm{au}$. This value is slightly larger than our determination, but in agreement within the error bars. In conclusion, the frequency of wide stellar companions to solar-type stars with or without planets (alt. close-in planets, radial velocity planets) seems similar whereas there might be an excess of wide substellar companions to planet-host stars, although this should be taken with caution because this determination is based on one case only.

\section{Dynamical properties planet-host stars}

In Fig. 5, we plot three diagrams depicting the period-mass, period-eccentricity, and mass-eccentricity relations for planethost stars in multiple systems (filled symbols) or isolated (open symbols). We have only included the 49 planet-host stars (open symbols) in the area in common between VHS and 2MASS. The planet-host stars with wide companions (filled black circles) are taken from Eggenberger et al. (2004) and Mugrauer et al. (2007a) and our new discoveries added as red dots with open squares. At the bottom right-hand side of Fig. 5 we have added a diagram showing the planet semi-major axis as a function of the binary projected separation of known planet-host stars from Mugrauer et al. (2007a) and our three new discoveries. We see a homogeneous distribution for the range shown. We observe that our survey fills the top right part of this diagram, extending the discovery to long periods and large binary separations with a special mention to the substellar companion.

Zucker \& Mazeh (2002) and Eggenberger et al. (2004) found two main trends in the period-mass diagram (top left panel in Fig. 5), facts supported by the enhanced number of multiple systems discussed in Mugrauer et al. (2007a). On the one hand, no stars with planets more massive than 2.5 Jupiter masses are found at periods less than 40 days. On the other hand, stars in multiple systems have planets less massive than $5 M_{\text {Jup }}$ for periods longer than 100 days. Our discoveries confirm the latter trend where the systems with the most massive planets exhibit periods longer than 950 days. We also found a wide companion to HD 126614, orbited by a small planet with a period longer than than 1000 days. However, the former trend does not seem to hold because over 80 short period ( $<40$ days) planets more massive than $2.5 M_{\text {Jup }}$ have now been announced around stars by several programs like the COnvection ROtation and planetary Transits (CoRoT) (Barge et al. 2006) and Kepler (Borucki et al. 2010) missions or the Hungarian-made Automated Telescope (HATNet; Bakos et al. 2002) and SuperWASP (Pollacco et al. 2006) projects, among others. These objects are not plotted in Fig. 5 because they lie outside the VHS coverage but half of them should be observed by the VHS during the next years because they are in the southern hemisphere. We note that only four of these stars with short-period planets more massive than $2.5 M_{\mathrm{Jup}}$ were known prior to 2007, at the time of Mugrauer et al. (2007a) publication. Moreover, most of these stars have small proper motions, typically less than 0.2 arcsec/yr (our lower limit for the astrometric search), which makes the search for co-moving companions extremely hard. This bias can be easily understood in the case of CoRoT, Kepler, HAT,Net and SuperWASP surveys, which are dedicated to the search for planetary transits without a specific requirement in proper motion.

We observe that the trend for low eccentricities and short periods among planet-host multiple systems holds (top right panel in Fig. 5) because we do not find any new candidate in this part of the diagram. We note that only one system, not included in the plots shown by Mugrauer et al. (2007a), has a short period ( $<40$ days) and an eccentricity higher than 0.05 , the possible planet "e" around 55 Cnc A (Wisdom 2005) which remains to be confirmed. We also find that our discoveries confirm the trend seen in the mass-eccentricity diagram (bottom left panel in Fig. 5), where more massive planets have on average higher mean eccentricities. Overall, we corroborate the conclusions of Desidera \& Barbieri (2007) that wide companions seem to have less influence on the properties of planets than closer systems.

\section{Conclusions and future work}

We identified new potential common proper motion wide companions to known stars harbouring extrasolar planets. We confirmed spectroscopically two $M$ dwarf and one mid-T dwarf companions sharing the same distance and proper motion as the planet-host star. One companion to 91 Aqr remains as a candidate until radial velocity is available. Moreover, four candidates lie further than 100000 au suggesting that these systems are not bound but might originate from a common stellar origin, share a similar dynamical evolution, or are disrupted binaries drifting 

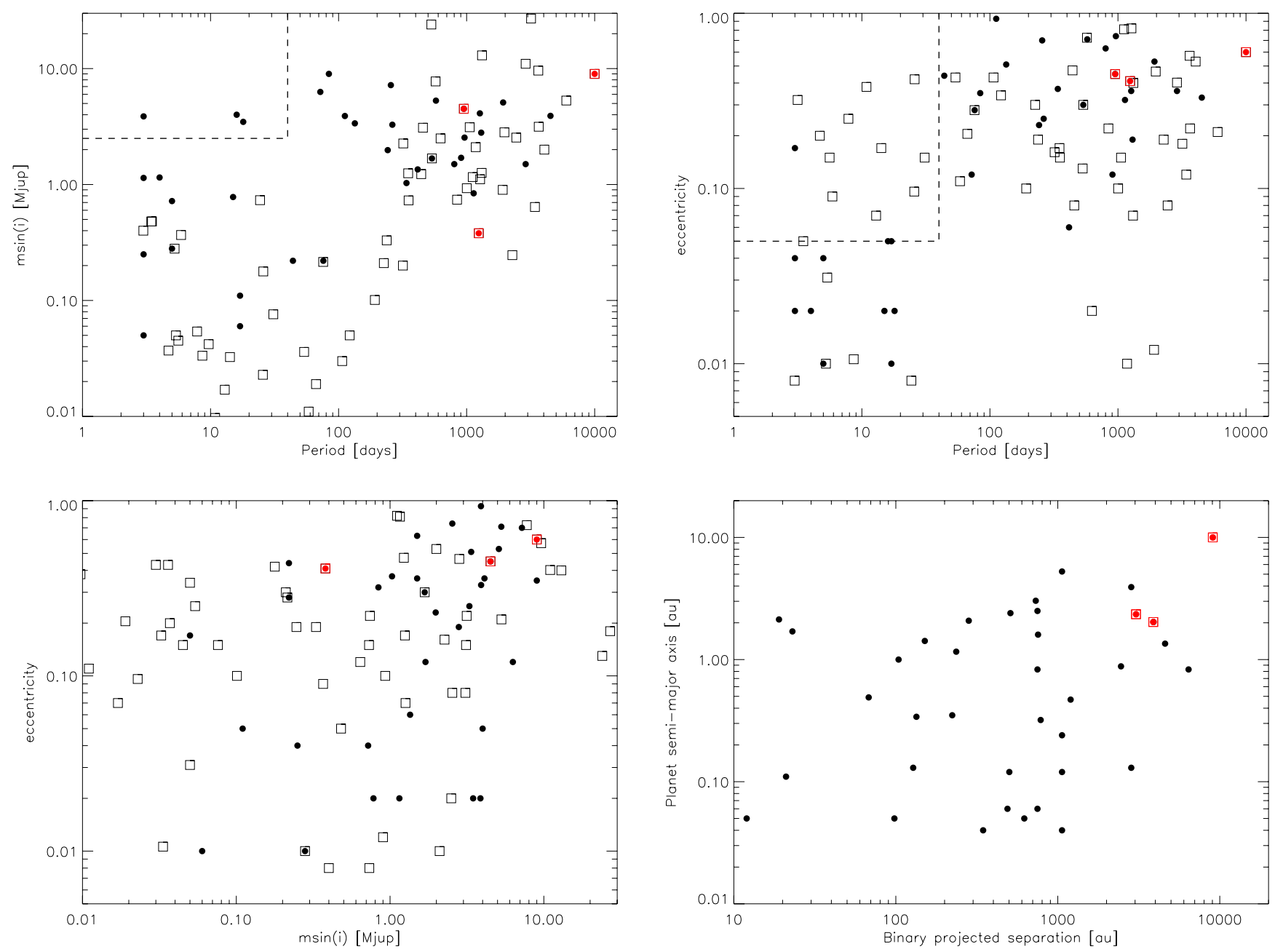

Fig. 5. Diagrams showing the period-mass (top left), period-eccentricity (top right), mass-eccentricity (bottom left), and binary projected separation vs. planet semi-major axis (bottom right) relations for single stars with planets within the VHS area considered in this work and multiple-star systems with planets (filled symbols). Black dots represent known planet-host stars previously published in the literature whereas red dots with open squares show our three best new discoveries. Plots amended from the original version published by Eggenberger et al. (2004) and later updated by Mugrauer et al. (2007a), the lower right plot being added.

away slowly from each other. The highlight of our search is the discovery of the second brown dwarf companion to a planet-host star, HIP 70849, which might be an equal-mass binary. Overall, our success rate in confirming wide common proper motion companions lies between $19 \%$ and $50 \%$. We derive frequencies of stellar and substellar wide companions to planet-host stars of $5.4 \pm 3.8 \%$ and $2.7 \pm 2.7 \%$, respectively.

Considering the success of our search for wide companions to planet-host stars in a few thousands square degrees of the southern sky imaged by the VHS, we plan to extend our automatic selection procedure to the entire southern sky at the end of the VHS lifetime. Moreover, we plan to apply our method to northern sources cross-matching UKIDSS (Lawrence et al. 2007) with 2MASS to provide a full view of the binarity among planet-host stars. Additional studies of these new systems should include high-resolution imaging with adaptive optics techniques to look for close companions and radial velocity or transit searches to detect any additional exoplanet(s) in those systems.

Acknowledgements. N.L. was funded by the Ramón y Cajal fellowship number 08-303-01-02. N.L. and V.J.S.B. are financially supported by the projects AYA2010-19136 and AYA2010-20535 from the Spanish Ministry of Economy and Competitiveness (MINECO), respectively. A.P.G. has been supported by Project No. 15345/PI/10 from the Fundación Séneca and MINECO under the grant AYA2011-29024. This work is based on observations collected at the European Southern Observatory, Chile, under programme numbers 090.C0791(A), 091.C-0862(B), and 092.C-0874. Based on observation obtained as part of the VISTA Hemisphere Survey, ESO progamme, 179.A-2010 (PI: McMahon). This work is based on observations (program GTC65-13B; PI Lodieu) made with the Gran Telescopio Canarias (GTC), operated on the island of La Palma in the Spanish Observatorio del Roque de los Muchachos of the Instituto de Astrofísica de Canarias. The VISTA Data Flow System pipeline processing and science archive are described in Irwin et al. (2004) and Cross et al. (2012). We have used data from the first data release (VHS DR1) described in McMahon (2012). We are grateful to the Cambridge Astronomy Survey Unit and the VISTA Science Archive at the Wide Field Astronomy Unit, Edinburgh, for providing us with the reduced data and catalogues. This research has made use of the Simbad and Vizier databases, operated at the Centre de Données Astronomiques de Strasbourg (CDS), and of NASA's Astrophysics Data System Bibliographic Services (ADS). This publication makes use of data products from the Two Micron All Sky Survey (2MASS), which is a joint project of the University of Massachusetts and the Infrared Processing and Analysis Center/California Institute of Technology, funded by the National Aeronautics and Space Administration and the National Science Foundation. This research has benefitted from the exoplanet.eu webpage and the SpeX Prism Spectral Libraries, maintained by Adam Burgasser at http://pono.ucsd.edu/ adam/ browndwarfs/spexprism

\section{References}

Bakos, G. Á., Lázár, J., Papp, I., Sári, P., \& Green, E. M. 2002, PASP, 114, 974 Barge, P., Léger, A., Ollivier, M., et al. 2006, in ESA SP 1306, eds. M. Fridlund, A. Baglin, J. Lochard, \& L. Conroy, 83 
Bertelli, G., Girardi, L., Marigo, P. \& Nasi, E. 2008, A\&A, 484, 815

Binney, J., \& Tremaine, S. 1987, Galactic dynamics (Princeton, NJ: Princeton University Press)

Bochanski, J. J., West, A. A., Hawley, S. L., \& Covey, K. R. 2007, AJ, 133, 531

Bonavita, M., \& Desidera, S. 2007, A\&A, 468, 721

Borucki, W. J., Koch, D., Basri, G., et al. 2010, Science, 327, 977

Boss, A. P. 2006, ApJ, 641, 1148

Burgasser, A. J. 2004, ApJS, 155, 191

Burgasser, A. J. 2007, ApJ, 658, 617

Burgasser, A. J., Kirkpatrick, J. D., Brown, M. E., et al. 1999, ApJ, 522, L65

Burgasser, A. J., Kirkpatrick, J. D., McElwain, M. W., et al. 2003a, AJ, 125, 850

Burgasser, A. J., McElwain, M. W., \& Kirkpatrick, J. D. 2003b, AJ, 126, 2487

Burgasser, A. J., Geballe, T. R., Leggett, S. K., Kirkpatrick, J. D., \& Golimowski,

D. A. 2006, ApJ, 637, 1067

Burningham, B., Pinfield, D. J., Lucas, P. W., et al. 2010, MNRAS, 406, 1885

Burningham, B., Cardoso, C. V., Smith, L., et al. 2013, MNRAS, 433, 457

Butler, R. P., Marcy, G. W., Williams, E., Hauser, H., \& Shirts, P. 1997, ApJ,

474, L115

Butler, R. P., Vogt, S. S., Marcy, G. W., et al. 2000, ApJ, 545, 504

Butler, R. P., Tinney, C. G., Marcy, G. W., et al. 2001, ApJ, 555, 410

Butler, R. P., Wright, J. T., Marcy, G. W., et al. 2006, ApJ, 646, 505

Buzzoni, B., Delabre, B., Dekker, H., et al. 1984, The Messenger, 38, 9

Cepa, J., Aguiar, M., Escalera, V. G., et al. 2000, in SPIE Conf. Ser., 4008, eds.

M. Iye, \& A. F. Moorwood, 623

Chanamé, J., \& Gould, A. 2004, ApJ, 601, 289

Chauvin, G., Lagrange, A.-M., Udry, S., et al. 2006, A\&A, 456, 1165

Chiu, K., Fan, X., Leggett, S. K., et al. 2006, AJ, 131, 2722

Close, L. M., Zuckerman, B., Song, I., et al. 2007, ApJ, 660, 1492

Covey, K. R., Ivezić, Ž., Schlegel, D., et al. 2007, AJ, 134, 2398

Cross, N. J. G., Collins, R. S., Mann, R. G., et al. 2012, A\&A, 548, A119

Cruz, K. L., Reid, I. N., Kirkpatrick, J. D., et al. 2007, AJ, 133, 439

Cutri, R. M., Skrutskie, M. F., van Dyk, S., et al. 2003, 2MASS All Sky Catalog of point sources, 2246

Dalton, G. B., Caldwell, M., Ward, A. K., et al. 2006, in SPIE Conf. Ser., 6269

Deacon, N. R., Liu, M. C., Magnier, E. A., et al. 2012, ApJ, 757, 100

Delfosse, X., Tinney, C. G., Forveille, T., et al. 1997, A\&A, 327, L25

Desidera, S., \& Barbieri, M. 2007, A\&A, 462, 345

Eggenberger, A., Udry, S., \& Mayor, M. 2004, A\&A, 417, 353

Eggenberger, A., Mayor, M., Naef, D., et al. 2006, A\&A, 447, 1159

Els, S. G., Sterzik, M. F., Marchis, F., et al. 2001, A\&A, 370, L1

Emerson, J. P. 2001, in The New Era of Wide Field Astronomy, eds. R. Clowes, A. Adamson, \& G. Bromage, ASP Conf. Ser., 232, 339

Emerson, J. P., Irwin, M. J., Lewis, J., et al. 2004a, in Optimizing Scientific Return for Astronomy through Information Technologies, eds. P. J. Quinn, \& A. Bridger, SPIE Conf. Ser., 5493, 401

Emerson, J. P., Sutherland, W. J., McPherson, A. M., et al. 2004b, The Messenger, 117, 27

Epchtein, N., de Batz, B., \& Capoani, L., et al. 1997, The Messenger, 87, 27

Fabricius, C., Høg, E., Makarov, V. V., et al. 2002, A\&A, 384, 180

Farihi, J., Becklin, E. E., \& Zuckerman, B. 2005, ApJS, 161, 394

Fischer, D., Valenti, J. A., \& Marcy, G. 2004, in Stars as Suns: Activity,

Evolution and Planets, eds. A. K. Dupree, \& A. O. Benz, IAU Symp., 219, 29

Fischer, D. A., Laughlin, G., Marcy, G. W., et al. 2006, ApJ, 637, 1094

Geballe, T. R., Knapp, G. R., Leggett, S. K., et al. 2002, ApJ, 564, 466

Ginski, C., Mugrauer, M., Seeliger, M., \& Eisenbeiss, T. 2012, MNRAS, 421, 2498

Gizis, J. E. 1997, AJ, 113, 806

Gontcharov, G. A. 2006, Astron. Lett., 32, 759

Gould, A., \& Chanamé, J. 2004, ApJS, 150, 455

Gould, A., \& Salim, S. 2003, ApJ, 582, 1001

Gray, R. O., Corbally, C. J., Garrison, R. F., et al. 2006, AJ, 132, 161

Haghighipour, N., Butler, R. P., Rivera, E. J., Henry, G. W., \& Vogt, S. S. 2012, ApJ, 756, 91

Hambly, N. C., Collins, R. S., Cross, N. J. G., et al. 2008, MNRAS, 384, 637

Hawley, S. L., Gizis, J. E., \& Reid, I. N. 1996, AJ, 112, 2799

Hog, E., Kuzmin, A., Bastian, U., et al. 1998, A\&A, 335, L65

Høg, E., Fabricius, C., Makarov, V. V., et al. 2000, A\&A, 355, L27

Houk, N. 1978, Michigan catalogue of two-dimensional spectral types for the HD stars

Howard, A. W., Johnson, J. A., Marcy, G. W., et al. 2010, ApJ, 721, 1467

Irwin, M. J., Lewis, J., Hodgkin, S., et al. 2004, in Optimizing Scientific Return for Astronomy through Information Technologies, eds. P. J. Quinn \& A. Bridger, Proc. of the SPIE, 5493, 411

Jameson, R. F., Casewell, S. L., Bannister, N. P., et al. 2008, MNRAS, 384, 1399

Jenkins, J. S., Jones, H. R. A., Goździewski, K., et al. 2009, MNRAS, 398, 911
Jenkins, J. S., Murgas, F., Rojo, P., et al. 2011, A\&A, 531, A8

Kirkpatrick, J. D., Looper, D. L., Burgasser, A. J., et al. 2010, ApJS, 190, 100

Kirkpatrick, J. D., Cushing, M. C., Gelino, C. R., et al. 2011, ApJS, 197, 19

Kirkpatrick, J. D., Gelino, C. R., Cushing, M. C., et al. 2012, ApJ, 753, 156

Knapp, G. R., Leggett, S. K., Fan, X., et al. 2004, AJ, 127, 3553

Koen, C., Kilkenny, D., van Wyk, F., \& Marang, F. 2010, MNRAS, 403, 1949

Konacki, M. 2005, Nature, 436, 230

Lagrange, A.-M., Beust, H., Udry, S., Chauvin, G., \& Mayor, M. 2006, A\&A, 459,955

Landolt, A. U., \& Uomoto, A. K. 2007, AJ, 133, 768

Lawrence, A., Warren, S. J., Almaini, O., et al. 2007, MNRAS, 379, 1599

Leggett, S. K., Allard, F., Dahn, C., et al. 2000a, ApJ, 535, 965

Leggett, S. K., Geballe, T. R., Fan, X., et al. 2000b, ApJ, 536, L35

Leggett, S. K., Marley, M. S., Freedman, R., et al. 2007, ApJ, 667, 537

Lépine, S., Rich, R. M., \& Shara, M. M. 2007, ApJ, 669, 1235

Liu, M. C., Leggett, S. K., \& Chiu, K. 2007, ApJ, 660, 1507

Lodieu, N., Pinfield, D. J., Leggett, S. K., et al. 2007, MNRAS, 379, 1423

Mace, G. N., Kirkpatrick, J. D., Cushing, M. C., et al. 2013, ApJS, 205, 6

Makarov, V. V., Zacharias, N., \& Hennessy, G. S. 2008, ApJ, 687, 566

Martín, E. L., Basri, G., Delfosse, X., \& Forveille, T. 1997, A\&A, 327, L29

Martín, E. L., Delfosse, X., Basri, G., et al. 1999, AJ, 118, 2466

Masciadri, E., Mundt, R., Henning, T., Alvarez, C., \& Barrado y Navascués, D. 2005, ApJ, 625, 1004

Mason, B. D., Wycoff, G. L., Hartkopf, W. I., Douglass, G. G., \& Worley, C. E. 2001, AJ, 122, 3466

Mayor, M., \& Queloz, D. 1995, Nature, 378, 355

McCarthy, C., \& Zuckerman, B. 2004, AJ, 127, 2871

McMahon, R. 2012, in Science from the Next Generation Imaging and Spectroscopic Surveys, id 37

Metchev, S. A., \& Hillenbrand, L. A. 2009, ApJS, 181, 62

Minniti, D., Butler, R. P., López-Morales, M., et al. 2009, ApJ, 693, 1424

Mitchell, D. S., Reffert, S., Trifonov, T., Quirrenbach, A., \& Fischer, D. A. 2013, A\&A, 555, A87

Montes, D., López-Santiago, J., Gálvez, M. C., et al. 2001, MNRAS, 328, 45

Moorwood, A., Cuby, J.-G., \& Lidman, C. 1998, The Messenger, 91, 9

Moriwaki, K., \& Nakagawa, Y. 2004, ApJ, 609, 1065

Mugrauer, M., \& Neuhäuser, R. 2005, MNRAS, 361, L15

Mugrauer, M., Neuhäuser, R., Mazeh, T., Alves, J., \& Guenther, E. 2004a, A\&A, 425, 249

Mugrauer, M., Neuhäuser, R., Mazeh, T., Guenther, E., \& Fernández, M. 2004b, Astron. Nachr., 325, 718

Mugrauer, M., Neuhaeuser, R., Guenther, E., \& Mazeh, T. 2005a, Astron. Nachr., 326, 629

Mugrauer, M., Neuhäuser, R., Seifahrt, A., Mazeh, T., \& Guenther, E. 2005b, A\&A, 440, 1051

Mugrauer, M., Neuhäuser, R., Mazeh, T., et al. 2006a, Astron. Nachr., 327, 321

Mugrauer, M., Seifahrt, A., Neuhäuser, R., \& Mazeh, T. 2006b, MNRAS, 373, L31

Mugrauer, M., Neuhäuser, R., \& Mazeh, T. 2007a, A\&A, 469, 755

Mugrauer, M., Seifahrt, A., \& Neuhäuser, R. 2007b, MNRAS, 378, 1328

Naef, D., Mayor, M., Lo Curto, G., et al. 2010, A\&A, 523, A15

Nakajima, T., Oppenheimer, B. R., Kulkarni, S. R., et al. 1995, Nature, 378, 463

Nelson, R. P. 2003, MNRAS, 345, 233

Nordström, B., Mayor, M., Andersen, J., et al. 2004, A\&A, 418, 989

O'Toole, S. J., Butler, R. P., Tinney, C. G., et al. 2007, ApJ, 660, 1636

Pancino, E., Altavilla, G., Marinoni, S., et al. 2012, MNRAS, 426, 1767

Patience, J., Ghez, A. M., Reid, I. N., \& Matthews, K. 2002, AJ, 123, 1570

Perryman, M. A. C., Lindegren, L., Kovalevsky, J., et al. 1997, A\&A, 323, L49

Pinfield, D. J., Burningham, B., Tamura, M., et al. 2008, MNRAS, 390, 304

Pollacco, D. L., Skillen, I., Collier Cameron, A., et al. 2006, PASP, 118, 1407

Poveda, A., Allen, C., Costero, R., Echevarría, J., \& Hernández-Alcántara, A. 2009, ApJ, 706, 343

Queloz, D., Mayor, M., Weber, L., et al. 2000, A\&A, 354, 99

Quinn, D. P., \& Smith, M. C. 2009, MNRAS, 400, 2128

Radigan, J., Lafrenière, D., Jayawardhana, R., \& Doyon, R. 2008, ApJ, 689, 471

Raghavan, D., Henry, T. J., Mason, B. D., et al. 2006, ApJ, 646, 523

Raghavan, D., McAlister, H. A., Henry, T. J., et al. 2010, ApJS, 190, 1

Rebolo, R., Zapatero-Osorio, M. R., \& Martín, E. L. 1995, Nature, 377, 129

Reid, I. N., Kirkpatrick, J. D., Liebert, J., et al. 1999, ApJ, 521, 613

Saffe, C., Gómez, M., \& Chavero, C. 2005, A\&A, 443, 609

Salim, S., \& Gould, A. 2003, ApJ, 582, 1011

Santos, N. C., Mayor, M., Naef, D., et al. 2001, A\&A, 379, 999 
Santos, N. C., Israelian, G., Mayor, M., et al. 2005, A\&A, 437, 1127

Santos, N. C., Sousa, S. G., Mortier, A., et al. 2013, A\&A, 556, A150

Scholz, R.-D., Kharchenko, N. V., Lodieu, N., \& McCaughrean, M. J. 2008, A\&A, 487, 595

Ségransan, D., Mayor, M., Udry, S., et al. 2011, A\&A, 535, A54

Shaya, E. J., \& Olling, R. P. 2011, ApJS, 192, 2

Skrutskie, M. F., Cutri, R. M., Stiening, R., et al. 2006, AJ, 131, 1163

Thompson, M. A., Kirkpatrick, J. D., Mace, G. N., et al. 2013, PASP, 125, 809

Tinney, C. G., Butler, R. P., Marcy, G. W., et al. 2002, ApJ, 571, 528

Tody, D. 1986, in SPIE Conf. Ser. 627, ed. D. L. Crawford, 733

Tody, D. 1993, in Astronomical Data Analysis Software and Systems II, eds. R. J. Hanisch, R. J. V. Brissenden, \& J. Barnes, ASP Conf. Ser., 52, 173
Torres, C. A. O., Quast, G. R., da Silva, L., et al. 2006, A\&A, 460, 695 van Altena, W. F., Lee, J. T., \& Hoffleit, E. D. 1995, The general catalogue of trigonometric (stellar) parallaxes van Leeuwen, F. 2007, A\&A, 474, 653

Vrba, F. J., Henden, A. A., Luginbuhl, C. B., et al. 2004, AJ, 127, 2948

Weinberg, M. D., Shapiro, S. L., \& Wasserman, I. 1987, ApJ, 312, 367

Wisdom, J. 2005, BA\&AS, 37, 525

Wittenmyer, R. A., Horner, J., Tuomi, M., et al. 2012, ApJ, 753, 169

Wright, J. T., Upadhyay, S., Marcy, G. W., et al. 2009, ApJ, 693, 1084

Wright, E. L., Eisenhardt, P. R. M., Mainzer, A. K., et al. 2010, AJ, 140, 1868

York, D. G., Adelman, J., Anderson, J. E., et al. 2000, AJ, 120, 1579

Zacharias, N., Urban, S. E., Zacharias, M. I., et al. 2003, UCAC2 Catalogue: $\mathrm{I} / 289$

Zucker, S., \& Mazeh, T. 2002, ApJ, 568, L113 


\section{Appendix A: Potential moving group members}

In this appendix, we describe the properties of the planet-host stars with very wide companions that might belong to moving group as well as systems rejected as bona-fide pairs based on our spectroscopic follow-up (Sect. 3; Table 1).

\section{A.1. BD 10-3166}

Butler et al. (2000) announced a planet with almost circular orbit $(e=0.05)$ and a mass of 0.48 Jupiter masses $\left(M_{\mathrm{Jup}}\right)$ with a period of $\sim 3.5$ days around the G4V solar-type star BD 10-3166 (Hog et al. 1998; Høg et al. 2000). The star is located at $66 \mathrm{pc}$ from the Sun, has an age of $\sim 4.2 \mathrm{Gyr}$, is metal-rich $([\mathrm{Fe} / \mathrm{H}]=0.5 \mathrm{dex})$, and has a proper motion of $\sim 0.2 \mathrm{arcsec} / \mathrm{yr}$. We confirm this estimated distance based on the absolute magnitude of a mid-G dwarf (e.g. Fig. 10 of Kirkpatrick et al. 2012).

We found a bright and wide common proper motion companion located at $\sim 39$ arcmin from BD 10-3166. We classify this potential companion as a M5-M5.5 dwarf based on the GTC optical spectrum. Using the $J$-band magnitudes of M5 and M5.5 dwarfs with their errors listed in Table 3 above, we derive a spectroscopic mean distance of 75-77 pc with a possible range of 63-90 pc. We conclude that these two objects are located at the same spectroscopic distance, with a projected physical separation of $\sim 150000-200000$ au.

\section{A.2. $H D 109749$}

Fischer et al. (2006) discovered a planet with a mass of $0.28 M_{\text {Jup }}$ with a period of $\sim 5.2$ days $(e=0.01$ ) around the G3IV (or G3V C) star, HD 109749 (Houk 1978). The planet-host star is located at $56.3 \pm 4.3 \mathrm{pc}$ with an age range of 7.4-13.2 Gyr and a metallicity estimated to $[\mathrm{Fe} / \mathrm{H}]=0.25 \pm 0.05$ dex. The primary is part of a double system formed by CCDM J12373-4049A and CCDM J12373-4049B, which are separated by $~ 8.4-9.2$ arcsec (Mason et al. 2001).

We detected two potential co-moving companions at 39.2 and 55.5 arcmin. We classify the closest wide companion as a K7 dwarf, comparing its spectrum with HD 157881 (Koen et al. $2010)^{9}$. Using G1825 as a K7V reference at $3.946 \pm 0.012 \mathrm{pc}$ (Hawley et al. 1996; van Leeuwen 2007), we determine a spectroscopic distance of approximately $140 \mathrm{pc}$. This distance estimate is 2.4 times greater than the distance of the planet-host star, implying that these two objects do not form a pair, even if we include the uncertainty on spectral classification and binary effects.

We classify the second companion as a M3.5 \pm 0.5 dwarf by direct comparison with Sloan templates. We derive a mean spectroscopic distance of $73.3 \mathrm{pc}$ for the potential companion, with a range of 45-144 pc including the uncertainties on the spectral type and absolute magnitude vs. spectral type relations. Hence, we conclude that these two objects are located at the same spectroscopic distance.

\section{A.3. $H D 143361$}

Jenkins et al. (2009) detected an eccentric planet $(e=0.17 \pm$ 0.15 ) with a mass of $3.12 M_{\text {Jup }}$ and a orbital period larger than 1000 days around HD 143361 (Houk 1978; Jenkins et al. 2011). The planet-host star is a G6V star (Houk 1978) at $65.66 \pm 5.51 \mathrm{pc}$ from the Sun (van Leeuwen 2007) and a

\footnotetext{
9 http://www .ast.obs-mip.fr/users/leborgne/stelib/ stars.html
}

metallicity of $\mathrm{Fe} / \mathrm{H}]=0.29$ dex (Nordström et al. 2004). We note that Minniti et al. (2009) quotes a spectral type of G0V (no reference is given though) and a distance of 59.35 pc for HD 143361 based on the early analysis of HIPPARCOs parallax (Perryman et al. 1997). Here we favour the new reduction of HIPPARCos data performed by van Leeuwen (2007).

We found two wide companions at 33.7 and 45 arcmin from the planet-host star. We classify the closest candidate as a M6M6.5 dwarf, suggesting a distance of 63-68 pc. Both components seem to be located at a very similar spectroscopic distance so we conclude that they might be physically associated. At the distance of 33.7 arcmin, we derive a projected physical separation of $\sim 120000-137000$ au for the pair.

We spotted a second potential co-moving companion at 45 arcmin from the planet-host star. We discarded that object photometrically. Nonetheless, we took an optical spectrum with NTT/EFOSC2 to confirm that it is an early-type star. Hence, this candidate companion is not associated with the planet-host star.

\section{A.4. $H D 159868$}

Wittenmyer et al. (2012) announced a second planet orbiting HD 159868 (Houk 1978) in addition to an eccentric planet candidate reported by O'Toole et al. (2007). After collecting more data and re-analysing the periodiograms of HD 159868, Wittenmyer et al. (2012) concluded that this subgiant star at $58.7 \pm 2.7 \mathrm{pc}$ harbours two planets with masses of 2.1 and $0.73 M_{\text {Jup }}$ and periods of about 1180 and 353 days, respectively. The age of HD 159868 is estimated to be 8.1 Gyr with solar composition.

We uncovered two potential wide companion to the planethost star, at about 40.7 and 42.3 arcmin. We classify the closest as a M3.0 \pm 0.5 dwarf and the furthest as a sdM0.5 subdwarf. For the former, we derive a mean spectroscopic distance of 130 (72175) pc, twice larger than the distance of the planet-host star.

For the latter we infer a spectroscopic distance of $96 \pm 12 \mathrm{pc}$ based on the parallax of LHS $536(44.6 \pm 5.3 \mathrm{pc}$; van Altena et al. $1995)$ and its spectral type (sdM0.5; Gizis 1997). This distance is much larger than the distance of the planet-host star. Moreover, we observe a metallicity difference between this candidate and the planet-host star.

To summarise, we reject both potential companions as comoving objects to the planet-host star HD 159868.

\section{A.5. $H D 207832$}

Haghighipour et al. (2012) discovered two jupiter mass planets orbiting the solar-metallicity $(\mathrm{Fe} / \mathrm{H}=0.06) \mathrm{G} 5 \mathrm{~V}$ star HD 207832 (54.4 \pm 2.7 pc; van Leeuwen 2007; Jenkins et al. 2011) by radial velocity. Their minimum masses are estimated to 0.56 and $0.73 M_{\text {Jup }}$, with orbital periods of about 162 and 1156 days, and eccentricities of 0.13 and 0.27 , respectively.

We unearthed a wide common proper motion companion to HD 207832, located at 38.6 arcmin. We classify it as a M6.5 dwarf, implying a distance of $48 \mathrm{pc}(42-50 \mathrm{pc})$.

We conclude that these two objects most likely form a pair with similar spectroscopic distances and a projected physical separation of $\sim 97000-132000 \mathrm{au}$.

\section{A.6. HD 216435}

Butler et al. (2006) announced a new planet orbiting HD 216435 , a G0V star at $32.62 \pm 0.42 \mathrm{pc}$ (van Leeuwen 2007) with an age of $5.27 \mathrm{Gyr}$ (Saffe et al. 2005) and a metallicity of $[\mathrm{Fe} / \mathrm{H}]=0.244$. The planet, detected by radial velocity, has a minimum mass 
of $1.26 M_{\mathrm{Jup}}$, low eccentricity $(e \sim 0.07)$, and a period of $\sim 1300$ days.

We discovered a potential wide companion with $J=$ $12.631 \mathrm{mag}$ at about 42 arcmin from HD 216435. We classify it as a M4-M4.5 dwarf. We derive a spectroscopic distance of 52-65 pc (41-82) for the companion, suggesting that these two objects are not physically associated.

\section{A.7. 91 Aqr}

Mitchell et al. (2013) published a $3.2 M_{\text {Jup }}$ planet orbiting the K0 giant, 91 Aqr. The planet-host star is located at $45.9 \pm 0.6 \mathrm{pc}$ (van Leeuwen 2007) with an age of $3.56 \pm 0.63 \mathrm{Gyr}$, and solar composition. This planet-host star has the largest proper motion of our sample $\left(\sim 0.37^{\prime \prime} / \mathrm{yr}\right)$. The planet has a low eccentricity $(e=0.027)$ and a orbital period of 181.4 days.

We found a wide companion located at 52.9 arcmin from 91 Aqr and a proper motion in good agreement with the planethost star (van Leeuwen 2007). We infer a spectroscopic distance of 63 (39-125) pc. Hence, we can not discard the pair as wide co-moving system, which would have a projected separation of $\sim 146000$ au. Radial velocity measurement are required to further assess the companionship. 